Abstracts*)

\title{
ISMD2014
}

\section{Tenth International Symposium on Molecular Diagnostics}

\author{
Medical University of Graz \\ Institute of Hygiene, Microbiology and Environmental Medicine \\ Clinical Institute of Medical and Chemical Laboratory Diagnostics
}

Austrian Society for Laboratory Medicine and Clinical Chemistry

Graz, May 29-31, 2014

Organizers

H.H. Kessler, M. Hübner, A.C. Haushofer, R.B. Raggam

Scientific Committee

A.C. Haushofer, H.H. Kessler, R.B. Raggam, B.I. Santner, R.E. Stauber

*)These abstracts have been reproduced directly from the material supplied by the authors, without editorial alteration by the staff of this Journal. Insufficiencies of preparation, grammar, spelling, style, syntax and usage are the authors' responsibility. 
Session New Technologies 1 (A1 - A6)

A1

\title{
Assessing laboratory performance for the molecular detection and determination of transplant-associated pathogens through international proficiency testing
}

\author{
Di Lorenzo C. ${ }^{1}$, Wallace P.S. ${ }^{1}$, Niesters H.G.M. ${ }^{2}$, Van Loon A.M. ${ }^{3}$ \\ ${ }^{1}$ Quality Control for Molecular Diagnostics, Glasgow, UK \\ ${ }^{2}$ University Medical Centre Groningen, Groningen, The Netherlands \\ ${ }^{3}$ University Medical Centre Utrecht, Utrecht, The Netherlands
}

Background: Viral infections pose significant complications in transplant patients resulting in high morbidity and mortality. A number of viruses are of particular concern in transplant patients, these include: human herpes virus 6 (HHV6), human cytomegalovirus (CMV) EpsteinBarr virus (EBV), human adenovirus (ADV), and the polyomaviruses JC (JCV) and BK (BKV). Sensitive and quantitative molecular methods have become an essential part of the diagnostic routine allowing accurate pathogen assessment prior to transplant and accurate quantitative monitoring after the transplant has been performed. The generation of reliable and reproducible quantitative test results is therefore of paramount importance in clinical transplant settings. However, the lack of suitable reference material or International Standards for some pathogens including HHV6, ADV, JCV, and BKV, makes comparison of results across laboratories rather difficult. In the absence of International Standards, Proficiency Testing (PT) can provide a valuable tool in support of standardization.

Objectives: To evaluate laboratory performance for the molecular detection and determination of transplant-associated pathogens through international PT programs.

Materials and methods: PT data obtained over the last five years were collected and collated. The data from each programme was analyzed to determine the performance of participants in the detection and quantitation of selected viral pathogens that cause clinical illness in transplant patients.

Results: In general, results on the PT panels showed a trend towards an improvement in performance. The majority of assays used by laboratories showed a high degree of sensitivity with a decrease in the percentage of false positive results. An improvement in the precision of quantitative assays was also observed. Interestingly, although an International Standard has recently been introduced for CMV and EBV, PT results showed that the use of these standards has not been widely adopted yet. Although an increase in the number of datasets reported in $\mathrm{IU} / \mathrm{ml}$ was observed, the majority of laboratories still reported results in copies/ml. Furthermore, no significant impact in the detection and quantitation of CMV and EBV has been observed since the introduction of the International Standards.

Conclusions: The design of PT programs allows comparison across multiple laboratories using numerous molecular technologies therefore supporting standardization particularly in areas where International Standards or reference materials are lacking.

\section{Total testing solutions provided by molecular diagnostics for pathogens detection}

\author{
Ginocchio C.C. \\ Global Microbiology Affairs, bioMérieux, Durham, NC, USA and Division of Infectious Disease Diagnostics, Hofstra North Shore-LIJ School of \\ Medicine, Hempstead, New York, USA
}

\begin{abstract}
Today's microbiology laboratories are facing many challenges from both a clinical and economic standpoint. They are challenged with increased testing demands, an aging workforce, decreased reimbursement, and budgetary cuts. Rapid accurate results are needed to improve patient outcomes, reduce length of stay, comply with antibiotic stewardship goals and save hospitals costs. To improve testing services and efficiency, and meet different clinical demands, microbiology laboratories need various solutions that combine automation and molecular diagnostics. This talk will discuss various molecular diagnostics testing formats available from bioMérieux and how automated platforms provide complete solutions for different clinical settings (out-patient, in-patient, emergency department, critical care settings) and different clinical needs (routine versus STAT testing, diagnostic versus monitoring). The incorporation of these platforms into the routine workflow will be presented. The concept of molecular diagnostics "Point of Care" testing versus "Point of Impact" testing will be discussed.
\end{abstract}

Rapid isothermal molecular diagnostics for infectious disease and hemato-oncology: DiaSorin Q-LAMP

Dolan A. 
Background: DiaSorin Q-LAMP is an isothermal, rapid, and highly specific nucleic acid amplification method designed for use on the LIAISON Iam system. DiaSorin has adapted loop-mediated isothermal amplification (LAMP), historically used for research, into an advanced form, Q-LAMP.

Objectives: To design, optimize, and validate rapid Q-LAMP diagnostic assays for infectious diseases and hemato-oncology.

Materials and methods: Q-LAMP utilizes five oligonucleotide primers and a fluorescent Q-probe, which recognize eight separate binding sites on the target nucleic acid. The method relies on auto-cycling strand displacement DNA synthesis, driven by the Q-LAMP polymerase in combination with specially designed primers.

Results: Amongst others, CE-IVD diagnostic assays for parvovirus B19 (Figure 1), BK virus and Toxoplasma gondii were developed and released with comparable performance to alternative amplification methods.

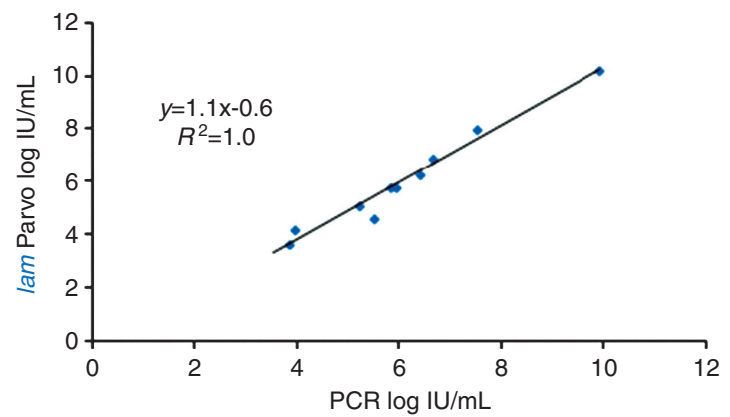

Figure 1 Quantitative correlation between lam Parvo Q-LAMP and PCR

Conclusions: Q-LAMP is a rapid diagnostic amplification method suitable for use in clinical laboratories. Single tube, single temperature RNA amplification, qualitative and quantitative applications for infectious disease and hemato-oncology are all features of Q-LAMP molecular diagnostics.

\section{A4}

\section{PCR coupled to mass spectrometry analysis for diagnosing viral infections in plasma samples of immunosuppressed patients}

Mengelle C. ${ }^{1}$, Le-Goff J. ${ }^{2}$, Mercier-Delarue S. ${ }^{2}$, Sauné K.. ${ }^{1}$, Mansuy J.-M. ${ }^{1}$, Simon F. ${ }^{2}$, Izopet J. ${ }^{1}$

${ }^{1}$ Department of Virology, University Hospital and INSERM U1043, CHU Toulouse, France

${ }^{2}$ Microbiology Laboratory, Saint-Louis University Hospital and INSERM U941, Paris Diderot University, Paris, France

Background: The PLEX-ID PCR coupled to electrospray-ionization mass spectrometry (PCR/ESI-MS) platform combines a broad-range PCR for viral nucleic acid detection.

Objectives: To evaluate the performance of the PLEX-ID PCR/ESI-MS for detection of viruses in the plasma of immunocompromised patients. Materials and methods: Whole blood samples underwent prospective analysis at the Department of Virology at the Toulouse University Hospital by routine monoplex PCRs for one or more of the following viruses: CMV, EBV, HSV-1, HSV-2, VZV, HHV-6, HHV-8, adenovirus, parvovirus B19, enteroviruses, and polyomaviruses BK and JC. Nucleic acids were extracted with the MagNA Pure 96 DNA and the Viral NA Small Volume kit ${ }^{\circledR}$ on the MagNA Pure $96^{\mathrm{TM}}$ (Roche Molecular Diagnostics, Meylan, France) instrument. Additionally, nucleic acids were extracted using the PLEX-ID total nucleic acid isolation kit. After extraction, batch analysis was performed on the PLEX-ID platform at the Microbiology Laboratory of the Saint-Louis University Hospital, Paris. Data were analyzed using Stata ${ }^{\mathrm{TM}}$ software 9.2 (StataCorp, Texas). The Kappa test was used whenever possible.

Results: 250 whole blood samples were collected from 216 immunocompromised patients, 11 children (median age, 5) and 205 adults (median age, 52). 480 routine tests were performed, 58 of which were positive with routine assays. With PCR/ESI-MS, 87 samples were found to be positive (107 pathogens) in single ( $n=68)$ or multiple $(n=19)$ infections, 139 negative, and 24 uninterpretable due to negative internal controls. Of 58 samples that tested positive with routine assays, PCR/ESI-MS detected 24 (41.4\%) pathogens, while 6 (10.3\%) samples gave uninterpretable results. Results correlated well for CMV, BKV, and EBV (Kappa tests 0.6 [0.5-0.8, 95\%], 0.9 [0.7-1.0, 95\%], and 0.6 [0.4-0.8; 95\%]). The CMV-positive $(n=8)$ and EBV-positive ( $n=15$ ) samples that gave discrepant results (positive by routine assay and negative by the PCR/ESI-MS) had low virus loads. With PCR/ESI-MS, 7 pathogens were detected in samples that were negative by routine assays and 63 pathogens in those samples that had not been tested by routine assays. Additionally, the PCR/ESI-MS detected 19 multiple infections (18 double infections and one triple infection), while routine assays detected 4 double infections. Finally, the PCR/ESI-MS detected 8 infections with HHV-7.

Conclusions: The multiplex format of PCR/ESI-MS makes it suitable for rapid and broad-range virological screening of immunocompromised patients at risk of developing severe viral infections. The PCR/ESI-MS can also detect uncommon viruses that may have a clinical impact in these patients. 


\title{
Electrospray-ionization mass spectrometry for bacterial and fungal detections in immunocompromised patients
}

\author{
Simon F. ${ }^{1}$, Le Goff J. ${ }^{1}$, Mercier-Delarue S. ${ }^{1}$, Hussenet C. ${ }^{2}$, Vérine J. ${ }^{3}$, Balsat M. ${ }^{4}$, Alanio A. ${ }^{1}$, Picard-Maureau M. ${ }^{5}$, Lafaurie-Bergeron A. ${ }^{2}$ \\ ${ }^{1}$ Microbiology and Mycology, ${ }^{2}$ Pneumology, ${ }^{3}$ Pathology, ${ }^{4}$ Hematology, Saint-Louis University Hospital and INSERM U941, Paris Diderot University, \\ Paris, France \\ ${ }^{5}$ Ibis/Abbott Molecular, Wiesbaden, Germany
}

Background: The PLEX-ID PCR coupled to electrospray-ionization mass spectrometry (PCR/ESI-MS) platform combines a broad-range PCR for bacterial and fungal DNA detection. Nucleic acids are extracted directly from the samples by a magnetic beads based method before broadrange PCR. The PCR-amplification products are characterized by electrospray ionization mass spectrometry. After MS analysis, the PLEX-ID software converts the mass information into base compositions. The PCR product base compositions are compared to base composition signatures in a database, allowing identifying multiple organisms within a sample. The results are obtained within 6 hours from DNA extraction to results reporting.

Objectives: To evaluate the accuracy of the Abbott PLEX-ID PCR/ESI-MS platform and assays in clinical samples negative in the routine microbiology procedures and in patients with clinical presentation suggestive of infections.

Materials and methods: In case of negative routine bacterial and fungal culture but clinical and biological evidences of an infectious ongoing process, nucleic acids were extracted from different samples including whole blood, plasma, BAL, biopsies and then analyzed with the PLEX-ID using broad-range bacterial and fungal assays (Abbott Molecular, Des Plaines, IL, USA; RUO). A medical panel with pathologists and respiratory-infectious diseases experts validated the PLEX-ID results with regard to the clinical and biological results.

Results: The PLEX-ID PCR/ESI-MS platform was found to successfully resolve different infections in immunocompromised patients when routine methods gave negative results: T. pallidum in testis, C. trachomatis in peritoneal fluid, A. naesludi, F. nucleatum, and H. capsulatum in pulmonary biopsies or BAL, U. urealyticum in synovial liquid, and F. solani in skin erythrosis were all considered as clinically relevant. C. albicans in a generalized candidiasis despite caspofungine treatment and the presence of different bacterial DNAs despite adapted antibiotics were also reported.

Conclusions: These clinical cases reported in immunocompromised patients infected with low or no growing pathogens in routine culture show evidence for advantages of using The PLEX-ID PCR/ESI-MS platform.

\section{A6}

\section{Performance characteristics of the Unyvero $^{\mathrm{TM}}$ system in the routine clinical microbiology laboratory}

König B.

Institute of Medical Microbiology and Infectious Epidemiology, Medical University of Leipzig, Leipzig, Germany

Background: Microbial culture is the current standard for the diagnosis of bacterial infections of the respiratory tract and takes at least 2 to 5 days to obtain diagnostic information. Currently available molecular methods focus only on a few pathogens and drug resistances. The nearly fully automated, commercially available Unyvero ${ }^{\mathrm{TM}}$ system (Curetis AG, Holzgerlingen, Germany) targets 17 pathogens, together with their resistance patterns, relevant in community-, hospital-, and ventilator-acquired respiratory tract infections.

Objectives: To show the efficiency of this system under study terms but not under routine terms in a university hospital.

Materials and methods: In total, 40 different respiratory samples were analyzed by culture in the routine setting at the Institute of Medical Microbiology at the university hospital in Leipzig as well as by the Unyvero ${ }^{\mathrm{TM}}$ system. All data were reported in a semiquantitative manner.

Results: Among the 40 samples, culture detected the presence of 1 pathogen in 20 samples, more than 1 pathogen in 13 samples, and no pathogen in 7 samples. The molecular analysis detected the presence of 1 pathogen in 10 samples, more than 1 pathogen in 27 samples and no pathogen in 3 samples. The direct comparison between culture and molecular diagnostics revealed that a) 3 samples were negative by molecular diagnostics and culture; b) in 10 samples, the number of pathogens detected and the type of pathogen were identical; c) in 26 samples, molecular diagnostics detected one or more additional pathogens. The pathogens detected additionally were Streptococcus pneumonia (11x), Staphylococcus aureus (8x), Haemophilus influenza (5x), Klebsiella spp. (3x), Escherichia coli (2x), Stenotrophomonas maltophilia (3x), Legionella pneumophila (1x), Pneumocystic jirovecii (1x), Pseudomonas aeruginosa (3x), Proteus spp. (1x), and Morganella morganii (1x). In 1 sample, molecular diagnostics missed Escherichia coli.

Conclusions: Data clearly show that the Unyvero ${ }^{\text {TM }}$ system a) detects all relevant pathogens; b) detects pathogens which might be overlooked easily; c) identifies the relevant pathogens approximately 2-3 days earlier as compared to culture. In addition to the identification of relevant pathogens, the Unyvero ${ }^{\mathrm{TM}}$ system provides the clinicians with the most important resistance patterns of the respective pathogens. 


\title{
Session New Technologies 2 (A7 - A12)
}

\section{A7}

\section{Low level virus load detection confidence of Abbott Real-Time HIV-1, Roche TaqMan HIV-1, and Versant HIV-1 RNA (bDNA) technologies for quantitation of plasma HIV-1 RNA in HIV-1 infected individuals}

\author{
Asthana D. ${ }^{1}$, Toth M. ${ }^{1}$, Bolivar H. ${ }^{2}$, Young T.P. ${ }^{3}$, Cloherty G.A. ${ }^{3}$, Valiathan R. \\ ${ }^{1}$ Department of Pathology, University of Miami, Florida, USA \\ ${ }^{2}$ Infectious Diseases-Internal Medicine, University of Miami, Florida, USA \\ ${ }^{3}$ Abbott Laboratories, Abbott Park, Illinois, USA
}

Background: Accurate and reproducible viral load is important in effective clinical and therapeutic management of HIV-1 infected individuals. Various in-vitro assays are available for measurement of HIV-1 RNA levels.

Objectives: To compare the Abbott Real-Time HIV-1, the Roche TaqMan HIV-1, and the Siemens Versant HIV-1 RNA bDNA assays in parallel on plasma specimens of 187 HIV-1 infected individuals. To assess the robustness of the assays by determination of low viral levels.

Materials and methods: Whole blood EDTA specimens were received at the Laboratory for Clinical and Biological Studies, University of Miami, FL for plasma HIV-1 RNA as part of an ongoing study. Quantitation of plasma HIV-1 RNA was performed in parallel using the three platforms according to approved protocols provided by the manufacturers. Statistical evaluations were carried out using Prism software (v 6.0). Results: Among 187 plasma specimens, HIV-1 RNA was detected in 153 specimens by Abbott (sensitivity, 82\%), 159 specimens by Roche (sensitivity, 85\%) and 150 specimens by Siemens (sensitivity, 80\%). The HIV-1 RNA levels obtained from all the three methods positively correlated with each other (Pearson Correlation, $p<0.0001$ ) indicating specificity of detection of each method. When comparing the technologies on a one to one basis, HIV-1 RNA levels obtained with Abbott and Siemens showed a higher linear association $(r=0.95)$ versus Abbott and Roche $(r=0.79)$ or Roche and Siemens $(r=0.85)$. However, when samples with $<100$ copies $/ \mathrm{ml}$ were compared, the three methods showed varying levels of detection and the detection levels did not correlate to each other. Comparison of the $95 \%$ confidence levels for the Abbott and Roche assays revealed 44 copies/ml (CI: 39.5-48.5 copies/ml) for Abbott and 52 copies/ml (CI: 48.0-56.7 copies/ml) for Roche.

Conclusions: All three assays showed an increasing variation of copy numbers with decreasing viral loads. This finding might influence the interpretation of the outcome of treatment based on virus suppression when a single assay is considered for evaluation. Results indicate that the lower detection threshold of assays might be influenced by factors associated with inherent variability of the assay technologies.

\section{A8}

\section{The cobas $® 6800 / 8800$ Systems: redefining molecular testing performance, flexibility, and automation}

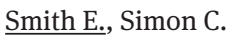

Roche Molecular Systems Inc., Pleasanton, California, USA

Background: The cobas ${ }^{\circledR} 6800 / 8800$ Systems were developed to address the changing needs in molecular testing, through the inclusion of unmatched testing flexibility, maximum walk away time, and the ability to rapidly generate test results. The molecular testing laboratory is tasked with performing a wide variety of molecular assays that currently require the use of different reagents and consumables. This requirement creates logistical constraints for laboratories and introduces the potential for errors in the testing process. Currently, no single molecular testing platform is able to offer the flexible testing capabilities that laboratories require (i.e. mixed batching of different sample types and required tests) while maintaining throughput, speed and quality assay performance.

Objectives: To develop the cobas omni process, a harmonized, universal sample preparation and thermal profile (PCR profile), that could be applied to all assays performed in an IVD lab without sacrificing assay performance.

Materials and methods: The initial portion of the presentation will discuss key areas of the sample preparation and PCR process where harmonization could occur. The latter portion of the presentation will focus on some of the strategies and techniques that were utilized to achieve assay performance while using a universal process and profile for diagnostic assays. The technical validation of the universal profile will be shown through demonstration of sensitivity in Blood Screening assay performance and the specificity and linearity associated with viral load assay performance.

Results: The results indicate a significant advancement in further driving automated platform performance. The cobas ${ }^{\circledR} 6800 / 8800$ Systems now have the ability to rapidly test samples, generating the first 96 results in under 3.5 hours. Through utilization of the cobas omni process, which maintains or improves assay performance from existing on-market assays, the cobas ${ }^{\circledR} 6800 / 8800$ Systems deliver testing flexibility. Users of these systems are now able to test up to 3 analytes in parallel quickly and with the highest throughput. The cobas ${ }^{\circledR} 6800$ System generates up to 384 test results in 8 hours while the cobas ${ }^{\circledR} 8800$ System generates up to 960 test results in the same time period. 
Conclusions: The cobas ${ }^{\circledR} 6800 / 8800$ Systems represent significant advancements in moelcular testing performance, flexibility, and automation. These advancements are based on the core foundation of the cobas omni process, which delivers robust assay performance and flexibility without sacrificing sensitivity or specificity.

\title{
A9
}

\section{A novel and rapid multiplex molecular sample-to-answer system for the detection of gastrointestinal pathogens}

\author{
Schroeder A.R.W., Tyler E.M., Leatham B.R., Harrel B.T., Van Sickle R.P., Freeman-Cook L.L.
} GenMark Diagnostics Inc., Carlsbad, California, USA

Background: Infectious diarrhea continues to be a global burden. GenMark's Gastrointestinal Pathogen Panel (GPP) is currently under development on a sample-to-answer platform, which will integrate sample extraction and amplification together with GenMark's proprietary eSensor ${ }^{\circledR}$ technology. The GPP assay is being designed to enable the concurrent detection of highly multiplex bacterial, viral, and parasitic organisms on a single test cartridge allowing for the rapid and comprehensive molecular detection of gastrointestinal (GI) pathogens.

Objectives: To investigate and assess the feasibility of a novel sample-to-answer platform for the detection of GI pathogens in contrived stool specimens.

Materials and methods: The performance of sample extraction and multiplex RT-PCR amplification was evaluated and compared to bench top methods. Model organisms including human adenovirus, norovirus GII, and Cryptosporidium were spiked into stool matrix. Samples were extracted using either the sample-to-answer system or the NucliSENS ${ }^{\circledR}$ easyMAG® system (BioMérieux, Durham, NC, USA). The purified nucleic acid was amplified in multiplex real-time RT-PCR (TaqMan $\left.{ }^{\circledR}\right)$ reactions to compare extraction yields between both methods. RT-PCR amplification was compared between on-cartridge and bench top methods. Both on-cartridge and bench top amplified targets were detected via capillary electrophoresis (CE) and GenMark’s proprietary eSensor ${ }^{\circledR}$ technology.

Results: The real-time RT-PCR results demonstrated similar extraction efficiencies between the sample-to-answer and the NucliSENS® easyMAG® system for the three targets under study. There was no statistically significant difference between the amount of PCR product produced on-cartridge and on bench top as determined by CE for the three targets (Tukey Analysis, JUMP v.9). Additionally, the PCR products were detected using GenMark’s proprietary eSensor ${ }^{\circledR}$ technology.

Conclusions: The results demonstrate successful sample-to-answer detection capability utilizing various elements of GenMark's unique sample-to-answer platform. This system will incorporate rapid, fully automated sample preparation and digital microfluidics to enable concurrent molecular detection of a broad range of targets on a single test cartridge. This platform is being designed to deliver rapid, sensitive and specific test results from stool, which would provide actionable results up to days sooner than traditional methods.

A10

\section{First results from the performance evaluation and workflow study of the Sentosa system - a new automated molecular platform}

Tiemann C. ${ }^{1}$, Knoop D. ${ }^{1}$, Hartmann A. ${ }^{1}$, Matthiesen P. ${ }^{2}$, Bozic M. ${ }^{3}$, Kessler H.H. ${ }^{3}$

${ }^{1}$ Labcon-OWL Analytic, Bad Salzuflen, Germany

${ }^{2}$ Vela Diagnostics GmbH, Hamburg, Germany

${ }^{3}$ Research Unit Molecular Diagnostics, IHMEM, Medical University of Graz, Austria

Background: The molecular diagnostics market is growing rapidly and the number of companies utilizing new tools increasing continuously. The automated instrument systems already established focus mainly on a few high volume assays or utilize a cartridge based format for smaller volume testing. The Sentosa system and corresponding applications could fit between these two market areas, providing flexibility, broad menu, and the opportunity to consolidate different assays on the same platform.

Objectives: To evaluate the performance and the workflow of the Sentosa system in a multicenter setting.

Materials and methods: Evaluation of test performance was done running the Sentosa quantitative CMV test in three different study sites using standardized diluted sample material from AcroMetrix. For the workflow study, the Sentosa Norovirus assay was tested in four consecutive runs with 24 norovirus samples each. Runs were started at 7:20, 9:45, 11:20, and 13:10 H on the same day. Samples were loaded on the Sentosa ${ }^{\mathrm{TM}}$ SX101 instrument for automated magnetic-bead based sample extraction and PCR setup. Real-time PCR took place in the vessels on a 72-well rotor-disc which had been heat sealed and manually transferred to the Rotor-Gene Q (RGQ) MDx platform. Sample information was transferred automatically from the Sentosa ${ }^{\mathrm{TM}}$ SX101 instrument to the RGQ. Settings for data interpretation and PCR profiles were pre-defined in the RGQ software.

Results: Evaluation of test performance revealed mainly concordant results at all study sites. When the workflow was evaluated, time to result was found to be $180 \mathrm{~min}$ per run with the final set of results being obtained at 17:00 $\mathrm{H}$. Time for system setup including reagent and disposable loading as well as barcode scanning was about 30 minutes. 
Conclusions: This first evaluation of the Sentosa system showed its potential to comply with diagnostic requirements in a molecular lab. Future implementation of improved software which guides reagent and sample handling and open mode protocols will stimulate clinical investigators' interests.

\title{
A11
}

\section{Molecular detection of pathogens in feces}

Greffrath U., Heckmann M., Steimer M.

Fast-track diagnostics, Junglinster, Luxembourg

Conventional culture in combination with microscopy is no longer the gold standard for the etiological diagnosis of diarrhea. PCR, immunoassays, and even new-generation sequencing are now realistic alternatives to conventional approaches. A range of PCR based approaches including real time (singleplex or multiplex), bead based and microarray, with or without nucleic acid extraction are more sensitive and faster than the conventional methods. There are novel technologies that process feces even more efficiently in development, for example test cartridges with automated sample homogenization, cell disruption, nucleic acid purification, which deliver up to 50-plex target detection using qPCR and result in less than 30 minutes. However, enhancements of gold standard real time PCR protocols, such as tubes or plates that contain lyophilized mastermix, in which the eluate just has to be added, are more useful and cheaper than all-in-one systems are. They are fast to set up and simple to run.

\section{A12}

\section{Evaluation of the RealStar ${ }^{\circledR}$ HEV RT-PCR Kit 1.0 by different diagnostic laboratories in Europe}

Rottengatter K., Hess M.

altona Diagnostics GmbH, Hamburg, Germany

Background: Hepatitis E virus (HEV) infection is probably the most common cause of acute viral hepatitis worldwide. Since its discovery in the early 1980s, the knowledge about the prevalence and relevance of HEV in human health increased significantly, in particular in the last decade. The specific and sensitive detection of HEV gains more and more importance to prevent infection of patients at risk. Recently, the RealStar ${ }^{\circledR}$ HEV RT-PCR Kit 1.0 has been developed.

Objectives: To evaluate the CE-IVD labeled RealStar ${ }^{\circledR}$ HEV RT-PCR Kit 1.0 by different laboratories in Europe.

Materials and methods: In Germany, the Institute for Laboratory and Transfusion medicine (Heart and Diabetes Center Nordrhein-Westfalen, University Hospital Bad Oeynhausen) screened minipools for the presence of HEV RNA. In France, the INSERM U1043, University of Toulouse, evaluated the assay using serial dilutions of $4 \mathrm{HEV}$-positive reference samples (genotypes 3a, 3c, 3e, and 3f).

Results: Both sides showed the usability of the RealStar® HEV RT-PCR Kit 1.0.

Conclusions: The new kit may serve as a highly sensitive and specific tool for molecular diagnostics of HEV.

\section{Session Pathogens 1 - Hepatitis Viruses, HIV (A13 - A16)}

\section{A13}

\section{Viral hepatitis and liver transplantation}

\author{
Graziadei I., ${ }^{1,2}$ \\ ${ }^{1}$ Department of Internal Medicine II, Medical University of Innsbruck, Austria \\ ${ }^{2}$ Department of Internal Medicine, District Hospital of Hall in Tyrol, Austria
}

Liver transplantation (LT) is the only curative therapeutic option for patients with end-stage liver disease. Hepatitis B virus (HBV) and hepatitis $\mathrm{C}$ virus (HCV) infection are among the most common causes of cirrhosis and hepatocellular carcinoma worldwide and a frequent indication for LT.

Although long-term treatment with nucleos(t)ide analogues (NUCs) results in stabilization and even improvement for most patients with decompensated HBV-induced cirrhosis and consequently reduce the need for LT, HBV-induced chronic liver failure still accounts for about $10 \%$ of all indications for LT. In the past, recurrence of hepatitis B after LT significantly reduced graft and patient survival. In the last two decades, the availability of HBV immunoglobulin (HBIg) and oral NUCs has facilitated the management of patients with hepatitis B by 
reducing recurrent infection and, consequently, resulting in excellent long-term outcome. Currently, combinations of HBIG and NUCs are considered the standard of care for prophylaxis against HBV recurrence after LT. However, economic pressure due to the high cost of HBIg prompted investigators to assess more cost-effective regimens, including lower doses of HBIg, intramuscular or subcutaneous injection or HBIg withdrawal in selected patients. In addition, the introduction of new NUCs with a greater antiviral potency and higher genetic barrier for viral resistance, tenofovir and entecavir, provides new options for patients undergoing LT for HBV-related liver cirrhosis. There is an ongoing debate regarding the optimal prophylactic strategy regarding efficacy and cost-effectiveness.

HCV infection is the leading cause of chronic liver disease and main indication for LT in the majority of European and North American centers. In contrast to HBV infection, recurrence of HCV infection is almost universal in all LT recipients. Recurrent infection may lead to accelerated progression of fibrosis with $20-30 \%$ of recipients developing graft cirrhosis within 5 years following LT. Recurrent HCV disease is thus the main cause of graft loss necessitating re-LT in the majority of patients. In addition, therapeutic approaches have achieved sustained virologic response in only one-third of treated patients and may cause severe side effects in more than half of patients. However, the rapid development of direct-acting antivirals against HCV will change the management of this disease and may prevent graft infection in the near future. Indeed, preliminary results have shown that interferon-free antiviral regimens are highly efficacious in the treatment of recurrent HCV disease. In addition, these treatments are safe with almost no side effects and drug interactions with immunosuppressive agents. Further results from larger studies, not only treating HCV patients after LT but also patients with decompensated liver cirrhosis prior to LT with the aim to avoid LT, are awaited eagerly.

\section{A14}

\section{Hepatitis E in developing and developed countries}

Izopet J.

Federative Institute of Biology, National Reference Center of Hepatitis E, INSERM U1043, Toulouse, France

Hepatitis E is a worldwide disease not restricted to developing countries. An improved understanding of the natural history of Hepatitis $\mathrm{E}$ virus (HEV) infection has been achieved within the last decade. Several reservoirs and transmission modes have been identified. HEV1 and HEV2 are transmitted between humans by contaminated water in areas of poor sanitation while in developed countries HEV3 and HEV4 are transmitted zoonotically from swine, wild-boar, deer, and rabbit. Transmission of HEV by transfusion seems also an important issue. Until recently, hepatitis E was underdiagnosed partly because of the use of low-sensitivity serological assays. However, diagnostics tools, including nucleic-acid tests, have been improved. The epidemiology and clinical features of hepatitis E differs between developing and developed countries. HEV infection is usually an acute self-limiting disease, but HEV3 infection causes chronic infections with rapidly progressive cirrhosis in organ-transplant recipients, hematological patients receiving chemotherapy, and individuals with HIV. HEV also causes extra-hepatic manifestations, such as neurological symptoms and kidney injury. Acute infection usually requires no treatment, but chronic infection should be treated by reducing immunosuppression in transplant-patients and/or the use of anti-viral therapy based on ribavirin. HEV infection can be prevented by improving the sanitary infrastructure mostly in developing countries and by avoiding eating undercooked pork or game meat. Several vaccine candidates have been developed. A recombinant vaccine based on virus-like particles obtained after expression of the capsid protein in $E$. coli is now available in China.

\section{A15}

\section{Proviral DNA as target of HIV-1 resistance analysis}

Lübke N. ${ }^{1}$, Oette M. ${ }^{2}$, Jensen B. ${ }^{3}$, Fätkenheuer G. ${ }^{4}$, Esser S. ${ }^{5}$, Lengauer T. ${ }^{6}$, Schülter E. ${ }^{1}$, Kaiser R. ${ }^{1}$, and the HIV-GRADE Study Team ${ }^{1}$ Institute of Virology, University of Cologne, Germany ${ }^{2}$ Department of Gastroenterology, Augustinerinnen Hospital, Cologne, Germany

${ }^{3}$ Department of Gastroenterology, Hepatology and Infectiology, University of Düsseldorf, Germany

${ }^{4}$ First Department of Internal Medicine, University of Cologne, Germany

${ }^{5}$ Department of Dermatology, University of Duisburg-Essen, Germany

${ }^{6}$ The Computational Biology and Applied Algorithmics Department, Max Planck Institute for Informatics, Saarbrücken, Germany

Background: HIV-1 resistance tests are routinely performed on the basis of viral RNA. As therapy failure, according to the EACS guidelines, is defined with a viral load of $>50$ copies $/ \mathrm{ml}$ and an accumulation of resistance mutations under failing therapy should be avoided, a change in medication in settings with low viral load is required. Because viral load is usually too low to perform a successful resistance test with HIV-1 RNA, resistance analysis with proviral DNA may provide an alternative.

Objectives: To evaluate HIV-1 resistance analysis based on proviral DNA.

Materials and methods: The plasma RNA and the corresponding proviral DNA of 80 EDTA samples of the RESINA cohort including 50 therapy naïve (TN) and 30 therapy experienced (TE) patients were isolated and analyzed for their resistance profiles in the protease (PR) and reverse transcriptase (RT) genes. 
Results: In 78 samples (TN=48, TE=30), analyzable sequences were obtained for both viral RNA and proviral DNA. In 39 samples, resistance mutations were detected ( 9 samples with PR mutations and 30 samples with RT mutations). Twenty-three (59\%) samples with resistance mutations showed an equal or a higher number of mutations in proviral DNA compared to viral RNA, whereas the remaining 16 samples (41\%) showed a lower number of mutations in proviral DNA. NRTI mutations showed the highest concordance of detected mutations in RNA and DNA (83\%). NNRTI mutations showed a concordance of $70 \%$ and PIs of $81 \%$ (not significant). Regarding specific amino acid positions, the frequency of resistance mutations was higher in the viral RNA, except position 70 in the RT gene and position 30 in the PR gene with mutations being detected more frequently in the proviral DNA. As expected, viruses of TE patients showed a higher number of resistance mutations compared to those of TN patients. Samples of TE patients displayed a higher number of mutations in viral RNA, while those of TN patients displayed a higher number of mutations in proviral DNA.

Conclusions: Sequencing of HIV-1 proviral DNA provides useful information. Comparison of viral RNA and proviral DNA results in a higher rate of resistance mutations in proviral DNA in TN patients. In TE patients, proviral DNA did not reveal the same quantity of information as viral RNA, but may be helpful in cases with an unsuccessful resistance analysis and/or no existing historic resistance data. Resistance analysis of proviral DNA can thus be supportive for the evaluation of resistance when viral load is low or undetectable in TE patients, in cases of unavailable historic resistance information, and for detection of transmitted drug resistance in TN patients.

\section{A16}

\section{Vascular endothelial dysfunction: the putative interface of cardiovascular risk factors affecting populations living with and without HIV in Sub-Saharan Africa}

Goswami N. ${ }^{1}$, Stelzl E. ${ }^{2}$, Kessler H.H. ${ }^{2}$, Nawrot T. ${ }^{3}$, De Boever P., ${ }^{3,4}$, Estambale B. ${ }^{5}$, Akoua-Koffi C. ${ }^{6}$, Strijdom, H. ${ }^{7}$

${ }^{1}$ Research Unit Gravitational Physiology and Medicine, Institute of Physiology, Medical University of Graz, Austria

${ }^{2}$ Research Unit Molecular Diagnostics, IHMEM, Medical University of Graz

${ }^{3}$ Centre for Environmental Studies, Hasselt University, Belgium

${ }^{4}$ Environment and Health Research, VITO NV, Belgium

${ }^{5}$ Jaramogi Oginga Odinga University of Science and Technology, Bondo, Kenya

${ }^{6}$ Department of Biology, Faculty of Medicine and Center of Research for Development, Alassane Ouattara University, Bouake, Côte d'Ivoire

${ }^{7}$ Division of Medical Physiology, Faculty of Medicine and Health Sciences, University of Stellenbosch, South Africa

Background: Vascular endothelial dysfunction (EDy) is an early precursor of cardiovascular disease. Early detection may provide an opportunity for clinical interventions aimed at reversing EDy. Few studies have investigated a possible correlation between HIV-1 infection and antiretroviral treatment (ART) at one side and EDy on the other in Sub-Saharan African (SSA) populations. Furthermore, no data is available on the cardiovascular effects of environmental factors in SSA populations.

Objectives: To investigate EDy as a putative common pathophysiological consequence of two societal/health challenges in SSA, namely emerging cardiovascular risk factors (HIV-1 infection, ART) and personal air pollution exposure.

Materials and methods: This study will be conducted on populations in three SSA countries (South Africa, Kenya, and Ivory Coast). Patients with HIV-1 infection not yet on first- line ART, patients with HIV-1 infection on ART, and subjects without HIV-1 infection will be investigated. Baseline investigations will include measurement of endothelial function (flow mediated dilatation), screening for diseases and smoking behaviour, and measurement of personal air pollution exposure. Epidemiological studies will be supported by animal and in vitro investigations, in which the direct vascular and endothelial effects of first line ART regimes will be explored by investigating vascular function and endothelial cell function.

Results: This study will provide novel data regarding the overall prevalence of EDy and effects of HIV-1 infection and ART on vascular endothelial function in SSA populations.

Conclusions: This study will be the first of its kind to investigate the proposed questions and compare the results obtained in populations of three distinct SSA countries.

\section{Session Pathogens 2 - Transfusion-Related Pathogens, Pathogens Relevant in the Immunosuppressed (A17 - A20)}

\section{A17}

\section{Recent developments in virus safety of blood components and plasma products in Europe}

Nübling C.M.

Section of Molecular Virology, Paul-Ehrlich-Institute, Langen, Germany

Safety testing of blood components and plasma products is a continuous exercise triggered by changes in epidemiology of pathogens, by recognition of potentially underestimated threats, and by steady improvement of screening tests. Plasma derivatives entering the European 
market undergo an evaluation process including virus safety assessment. In contrast, cellular blood components are regulated on the national level, reflecting regional opportunities and needs. Estimation of virus safety of plasma derivatives includes residual risk calculation based on donor epidemiology, screening algorithms, and worst case assumptions. The state of art of screening assays is characterized by continuous improvement. Evolution of viral variants is a steady challenge for screening assays, especially NAT assays. The unexpected appearance of certain HIV-1 variants led to new requirements for the design of HIV-1 screening NATs on the national level (use of selected assays in Germany). Similar developments are expected on the European level in near future (CE-marking process). The recognition of WNV as a pathogen appearing increasingly in European regions led to safety measures in these regions, but also for travelers returning from regions affected. Standardization of WNV NATs remains an issue. HEV as an old, but newly recognized pathogen may be another potential threat to the blood supply; however, the implication of HEV for recipients of blood components still needs to be clarified.

\section{A18}

\section{HBsAg, HBV DNA, and anti-HBc: Which screening algorithm has the highest impact on blood safety?}

Chudy M., Kress J., Nübling C.M.

Section of Molecular Virology, Paul-Ehrlich-Institute, Langen, Germany

HBV safety of blood components is still an issue. The probability of HBV transmission by blood components in Germany is 10 times higher compared to transmission with HCV and HIV. The worldwide most essential HBV screening marker for blood donations is HBsAg. To further close the window period and/or to detect low-level chronic carriers, including donors with occult HBV infection (OBI), additional testing for HBV DNA and/or anti-HBc, respectively, is performed by some countries, depending on the epidemiology. HBsAg is the principal marker for diagnosis of HBV infection. It is the main component of the envelope of HBV particles and the subviral particles which can be secreted in large excess over the virus. There is no overall correlation between HBsAg and HBV DNA during the course of HBV infection. The analytical sensitivity between the different HBsAg test kits varies considerably. Only individual donation testing by NAT versus mini-pool NAT will have a measurable impact on the shortness of the window period compared to the screening with the most sensitive HBsAg tests. However, HBV genotype dependent secretion of subviral particles should be taken into consideration as described e.g. for genotype G. The availability of the WHO international standards for HBsAg and HBV DNA for more than 10 years is the prerequisite of the high standardization grade of the current diagnostic assays. Additionally, established HBV genotype international reference panels for both, HBsAg and NAT-based assays are necessary tools for the test evaluation in respect to the detection efficiency of different genotypes. Although anti-HBc is not an infectivity marker its suitability to detect HBV at different stages of the HBV infection is evident. Currently available anti-HBc tests validated with the recently established WHO international standard decreased the ratio of potential false positive reactions, which caused problems in particular in low prevalence HBV countries. Due to the lack of confirmatory tests, a special algorithm must be implemented to allow the re-entry of donors with unspecific repeat-reactive anti-HBc screening results. Nevertheless, anti-HBc screening of blood donations in HBV low endemic areas in addition to HBsAg and/or HBV NAT testing allows the identification of HBV low-level carriers including OBI donors.

A19

\section{Any news in testing transfusion transmitted infectious diseases?}

Gabriel C.

Red Cross Transfusion Service for Upper Austria, Linz, Austria

Transfusion medicine evolves out of blood donation, component production, and testing into new fields of interest, primarily into cell therapies and tissue engineering. It interfaces with nearly all clinical disciplines, but is heavily influenced by regulations of pharmaceutical quality systems. Some ever-lasting topics in transfusion transmitted infectious diseases (TTID) and molecular testing have still to be elucidated.

Emerging viruses: Recent discoveries were successful in detecting emerging viruses, which may bear risks for transfusions. But in the case of Xenotropic Murine Leukemia Virus-Related Virus it was shown that a more stringent decision making process would be better in transfusion medicine. This includes also the interpretation of epidemiological data for the introduction of new assays in Central Europe. As a best example, West Nile Virus NAT remains still confusing.

Sensitivity: Currently there are not so many, as otherwise expected, rules governing the use of NAT in TTID. Of growing concern is the level of sensitivity required and how these levels may reduce risks.

Barriers: Donor questioning avoids in many cases infections, which are avoidable. Currently there are growing political tendencies from minorities to circumvent donor eligibility restrictions due to anti-discrimination issues.

New tests: More and more specific infectious disease testing is needed for specific patient groups including herpes viruses, hepatitis $\mathrm{E}$ virus or parvovirus B19. Additional testing is required for plasma products and introduced in blood banks, too. 
Cadaveric donors: Tissue banking is evolving and standardization of extraction methods and tests remain as open questions. More data relating to physiological processes after death and their influence on the inhibition of samples are needed. There is currently no standard for tissue banking.

In conclusion, TTID testing seems to be a quiet area in transfusion medicine. Many issues are not solved yet. Many more decisions should be made in this field

\section{A20}

\section{Karolinska Institute and Washington University polyomaviruses: possible human pathogens?}

\section{Ciotti M.}

Laboratory of Molecular Virology, Polyclinic Tor Vergata Foundation, Rome, Italy

Background: For some decades, BK and JC polyomaviruses have been the only known members of the Polyomaviridae family of viruses of human origin. From 2007 to date, 10 novel human polyomaviruses have been discovered using advanced molecular techniques: KIPyV, WUPyV, MCPyV, HPyV6, HPyV7, TSPyV, HPyV9, HPyV10, STLPyV, and HPyV12. Disease association has been established for four of them: BKPyV, JCPyV, TSPyV, and MCPyV.

Objectives: To evaluate the pathogenic role of KIPyV and WUPyV in immunocompromised patients.

Materials and methods: 153 plasma samples from HIV-1 positive patients and 121 from 26 stem cell transplant recipients were screened for KIPyV and WUPyV DNAs by amplification of VP1 and LTAg genes. The amplification products were sequenced followed by phylogenetic analysis. In the HIV-1 infected patients, CD4+ cell counts and HIV-1 viral load were correlated to the frequency of infection with KIPyV and WUPyV. For the two study groups, 130 healthy blood donors and 26 bone marrow donors were enrolled as controls, respectively.

Results: In 153 HIV-1 positive patients, KIPyV DNA was detected in 4 (2.6\%) and WUPyV DNA in 7 (4.6\%) patients. Of 130 healthy blood donors, $4(3.1 \%)$ and $1(0.8 \%)$ were positive for KIPyV DNA and WUPyV DNA, respectively. No association was observed when CD4+ cell counts and viral loads were correlated to the frequency of infection with KIPyV or WUPyV. Phylogenetic analysis did not suggest circulation of specific KIPyV and WUPyV strains in HIV-1 positive patients. All plasma samples obtained from stem cell transplant recipients were negative for KIPyV and WUPyV DNAs. However, KIPyV DNA was detected in 2 bone marrow donors. There was no evidence of KIPyV transmission from the donor to the recipient. Conclusions: This data exclude an active role for KIPyV and WUPyV in HIV-1 positive patients as well as in the post-transplant clinical course of stem cell transplant recipients.

\section{Session Pathogens 3 - HPV, Other Viruses (A21 - A24)}

\section{A21}

\section{HPV diagnostics in clinical routine}

Ikenberg $\mathrm{H}$.

Cytomol, MVZ for Cytology and Laboratory Diagnostics, Frankfurt/Main, Germany

Since more than a decade molecular detection of High-Risk-(HR)-HPV has become a relevant element in the prevention of cervical cancer. Within recent years, large studies of high methodic quality have further underlined the importance of HPV clinical diagnostics. Studies demonstrated that for the detection of high-grade cervical preneoplastic lesions (>CIN2) HR-HPV DNA testing is more sensitive but less specific than cytology and that HPV DNA testing is less variable than cytology. It is of paramount importance that women with a negative HR-HPV DNA basic test have a significantly lower risk of developing a lesion $>$ CIN 3 than those with a normal cytology report.

There are three main fields where HPV molecular diagnostics is already in routine clinical use: 1) triage of cytological borderline and low grade abnormalities, 2) follow-up of patients after therapy of CIN as a test of cure, and 3) as an adjunct to conventional and thin layer cytology in women above 30 years. All these indications are recommended by US and European guidelines. Meanwhile, in some countries, even the substitution of cytology as primary screening instrument by HPV molecular testing is under discussion.

HPV prevalence and elimination rate are high, especially in young women. Only HPV persistence over a certain period is of clinical importance. It is of paramount importance to achieve high sensitivity and specificity for CIN 2+ standardized methods for HPV DNA detection. The balance between analytical (low) and clinical (high) sensitivity is crucial for the specificity of a routine HPV DNA test, especially in a screening approach. That is the reason why for a long time the HC2 test (Qiagen) was the only assay qualified for HPV DNA routine diagnostics.

New tests for routine HPV molecular testing must be validated according to a guideline defined by leading experts in the field. Meanwhile, several systems have passed this hurdle. Three of them, a signal amplification test (Cervista, Hologic), a qPCR-based test (cobas, Roche Diagnostics), and an RNA-based test (Aptima, Hologic) have received an FDA approval. Currently, the broadest data base is available for the cobas test, with the technique having been analyzed in the to-date largest HPV study (47000 participants).

HPV 16 and 18 implicate an up to 5x higher risk for the development of a CIN 2+ as other HPV HR types. It is therefore appropriate to test for these HPV types independently in parallel or after a positive HPV high-risk test. At present, a more detailed HPV typing is not useful in 
routine. HPV RNA testing may be an interesting option with probably higher specificity. Its clinical value still needs to be confirmed in longterm studies.

Even in the case of HPV 16/18 positivity, the large majority of lesions will not progress to cancer. Therefore, progression markers are valuable tools. Up to now, the best validated test is the combined immunocytochemical detection of p16 and Ki-67 in the same cell (CinTec PLUS, Roche MTM).

A22

Human papillomavirus infection and vaccination

Tabrizi S.N. ${ }^{1,2,3}$ on behalf of the VIP study group

${ }^{1}$ The Royal Women's Hospital, Melbourne, Australia

${ }^{2}$ Department of Obstetrics and Gynaecology, University of Melbourne, Australia

${ }^{3}$ Murdoch Childrens Research Institute, Melbourne, Australia.

Background: Cervical cancer is one of the few human cancers with well-defined link to a virus, i.e. human papillomavirus (HPV). In 2007, Australia introduced a funded quadrivalent HPV vaccination program supported by a national register. Between 2007-2009, school-based component achieved coverage of $83 \%$ and $70 \%$ for one and three doses respectively in girls aged 12-17, and community-based provision mainly via general practitioners to women aged $18-26$ achieved $55 \%$ and $32 \%$.

Objectives: To evaluate program impact on HPV prevalence in young women attending family planning clinics.

Materials and methods: Cervical HPV genoprevalence in women aged 18-24 years attending participating clinics for a Pap test in the prevaccine period (2005-2007) was compared with prevalence in the post vaccine period (2010-2011). With appropriate consent, vaccination status was confirmed against the HPV Register.

Results: Overall 1199 women were recruited for the post vaccine surveillance, of which $90 \%$ gave consent to access the register, with $50.2 \%$ fully vaccinated. The prevalence of any vaccine genotype $(6,11,16,18)$ was significantly lower in the post-vaccine sample than in the prevaccine sample $(7.0 \%$ versus $28.7 \%, p<0.001)$, and was associated with vaccination status in the post-vaccine sample (fully $2.8 \%$, partial/ unconfirmed $7.7 \%$, unvaccinated $20.4 \% ; p<0.001$ ).

Conclusions: Five years after the commencement of the Australian HPV vaccination program, a substantial fall in vaccine-targeted genotypes is evident.

A23

Prevalence of high risk human papillomavirus infections and associations with other sexually transmitted infections in HIV positive men who have sex with men

Ginocchio C.C.

Division of Infectious Disease Diagnostics, Hofstra North Shore-LIJ School of Medicine, Hempstead, New York, USA

Human papillomavirus (HPV) is the major etiological agent for ano-genital cancer. Although anal cancer is uncommon, there has been a significant rise in men who have sex with men (MSM) with the incidence in HIVpositive MSM the highest. The association of high risk (HR) HPV infection, anal cancer with a variety of other sexually transmitted infections (STIs) including Chlamydia trachomatis (CT), Neisseria gonorrhoeae (NG), Trichomonas vaginalis (TV), and Mycoplasma genitalium (MG) in HIV-1 positive men was studied in four US cohorts located in New York and California. Specimen types tested (1-3 per subject) included urine for CT, NG, TV, MG, throat for CT, NG, TV, MG, HR-HPV and rectal for CT, NG, TV, MG, HR-HPV. HPV genotyping for HPV 16, 18/45 was performed. In the NY cohort, anal cytology and histology, when indicated, was additionally evaluated. This lecture will present the cohort data in association with patient demographic considerations, and clinical parameters including HIV-1 RNA levels and CD4 T cell counts.

\section{A24}

\section{Rapid intra-individual norovirus evolution in chronically infected transplant recipients}

Hoffmann D., Haase K., Beck R., Avettand-Fenoel V., Zuber J., Frishman D., Protzer U. Institute of Virology, Technical University Munich/Helmholtz Center, Munich, Germany

Background: Chronic norovirus infection has been described recently and will become more prevalent with increasing numbers of immunocompromized transplant recipients.

Objectives: To study norovirus evolution of GII.4 strains during chronic infection in 8 patients after bone marrow and solid organ transplantation with next generation sequencing (NGS). 
Materials and methods: Two to five longitudinal specimens obtained from 8 chronically infected transplant recipients were investigated. Specimens covered a period of 38 to 470 days. Amplification products comprising 760 nucleotides and labeled with barcodes on both ends served as templates for sequencing. Consensus sequences of all samples were used for genotyping and multiple sequence alignment, including phylogenetic analysis.

Results: Norovirus Hu/NoV/Farmington Hills/2002/USA was chosen as reference strain for initial mapping. Consensus sequences could be mapped in $>95 \%$ to this reference sequence. Their evolutionary rate varied with up to 22 longitudinal amino acid mutations. Positive selection was determined by $\mathrm{dN} / \mathrm{dS}$ analysis in most time intervals, particularly at the end of infection. All consensus sequences evolved faster on amino acid than on nucleic acid level indicating positive selection. The number of reconstructed quasispecies first increased and then decreased before the infection was cleared.

Conclusions: This data illustrate the fast evolution of noroviruses under positive selection during chronic infections. NGS can monitor the complex changes of intra-individual viral populations. Understanding these processes may help in establishing therapeutic and prophylactic strategies including vaccines.

\section{Special Seminar (A25)}

\section{A25}

\section{Quality assurance in molecular diagnostics: practical approaches for addressing day-to-day challenges in a clinical laboratory}

Asthana D. ${ }^{1}$, Toth M. ${ }^{1}$, Valiathan R. ${ }^{1}$, Rathore M. ${ }^{2}$

${ }^{1}$ Department of Pathology, University of Miami, Florida, USA

${ }^{2}$ University of Florida - School of Medicine, Jacksonville, Florida, USA

Molecular diagnostics has become a powerful tool in the management of infectious diseases. Technology has successfully been used in the detection and quantitation of infectious agents, in rational administration of antibiotics and antiretrovirals, and in the identification of mutations specific to drug resistance. In addition, molecular diagnostics allows genotyping of viral diseases and screening blood donations for viral infections. Real-time PCR (qPCR), gene rearrangement analysis, and other nucleic acid-based microbial detection methods once considered to be only research tools are being used increasingly in the clinical laboratory. With the advent of standardized quantitative kits and the increase in quantitative in-house developed ("home-brew") testing, guidelines for the development, verification, assessment, and implementation of these assays are warranted along with quality assurance. The current routes of molecular detection of infectious diseases are progressing towards high-throughput, simple, array-type technologies that will provide worthy data on types of the causative agent and the virulence factors/resistance determinants which might influence the disease severity. Future of the molecular diagnostics of infectious diseases will certainly focus on a marked increase in the information detected with platforms that will simplify analysis of the data for rapid use in clinical decision making. However, several issues will continue to be discussed including sensitivity and specificity of the molecular technique to a wide variety of settings, guidelines towards performing and reporting quality results, interpretation of results, and issues pertaining to the implementation of internal and external quality controls. To achieve measurable quality improvement, a laboratory must be able to detect errors, implement system improvements, and measure outcomes. Participants will learn practical approaches to quality improvement using "real-life" laboratory situations and group discussion.

\section{Poster Session (P1 - P11)}

\section{P1}

\section{Prenatal RHD genotyping: in-house method validation}

Bingulac-Popović J. ${ }^{1}$, Đogić V. ${ }^{1}$, Babić I. ${ }^{1}$, Hundrić-Hašpl Ž. ${ }^{2}$, Mišković B. ${ }^{3}$, Mratinović-Mikulandra J.4, Juraković-Lončar N. ${ }^{2}$, Balija M. ${ }^{5}$, Jukić I. ${ }^{5}$ ${ }^{1}$ Department of Molecular Diagnostics, CITM, Zagreb, Croatia ${ }^{2}$ Department of Immunohematology, CITM, Zagreb, Croatia

${ }^{3}$ Department of Gynecology, Sveti Duh University Hospital, Zagreb, Croatia

${ }^{4}$ Department of Transfusion Medicine, Split University Hospital Center, Split, Croatia

${ }^{5}$ Transfusion Medicine Service, CITM, Zagreb, Croatia

Background: The presence of fetal DNA in maternal plasma and highly sensitive qPCR methods have enabled the noninvasive determination of the fetal Rhesus $\mathrm{D}(\mathrm{RhD})$ genotype in RhD-negative mothers to assess the risk of hemolytic disease in the newborn, the requirement of stringent pregnancy follow up, and the possible need for antenatal immunoprophylaxis. 
Objectives: To validate an in-house method of fetal $R H D$ genotyping in maternal plasma samples and to compare the prenatal RhD genotype with the postnatal RhD status.

Materials and methods: Validation included $24 \mathrm{RhD}$-negative women, gestation week 10 to 37, followed up at hospitals in Zagreb and Split. Fetal DNA was isolated from maternal plasma by manual procedure from $1000 \mu \mathrm{L}$ EDTA blood on Qiagen columns (Qiagen, Germany). Fragments of exons 7 and 10 of the RHD, SRY and RASSF1A genes, which served as fetal DNA markers, were amplified by qPCR on the AB 7500 real time (RT) PCR System (Applied Biosystems, USA). The RASSF1A marker was submitted to cleavage with the BsTUI restriction enzyme, with the noncleaved hypermethylated portion representing the fetal DNA marker. All samples were tested in triplicate over 3 days from isolation to qPCR. Results: Method validation indicated the optimal maternal plasma sampling after week 12 of gestation. Comparison with the postnatal RhD status showed $100 \%$ concordance with the prenatally determined $R H D$ genotype.

Conclusions: Fetal RHD genotyping helps to avoid unnecessary immunoprophylaxis in about 40\% of RhD-negative mothers carrying RhDnegative fetuses. Noninvasive exon 7 and exon 10 testing proved to be a robust method of fetal RHD determination.

P2

\section{Novel pharmacogenetic test for management of antiviral therapy in patients with chronic hepatitis C}

Bugin E. ${ }^{1,2}$, Cavalletto L. ${ }^{3}$, Bortolozzo K. ${ }^{1}$, Schiavo I., ${ }^{1,2}$, Chemello L. ${ }^{3}$

${ }^{1}$ AB ANALITICA srl, Padova, Italy

${ }^{2}$ Department of Pharmaceutical Sciences, Padova University, Padova, Italy

${ }^{3}$ Department of Medicine-DIMED, Padova University Hospital, Padova, Italy

Background: The association between host (IL28B/ITPA SNPs) and viral (genotype and kinetic) factors is important for HCV eradication by antiviral therapy. Recently, a pharmacogenetic test (GENEQUALITY IL28B-ITPA TYPE, AB ANALITICA) that combines multiplex PCR with reverse line blot hybridization for detecting IL28B (rs12979860 C > T, rs8099917 T>G) and ITPA (rs7270101 A>C, rs1127354 C>A) SNPs was developed.

Objectives: To identify patients with favorable profile of response or mild side effects by pharmacogenetic analysis.

Materials and methods: 340 patients ( $46 \pm 10$ years) with chronic hepatitis C were treated with Peg-IFN+RBV in real-life practice and tested with the newly developed assay.

Results: Patients with HCV genotype 1 or $4(n=186)$ showed a more advanced liver fibrosis than patients with HCV genotype 2 or 3 ( $n=154)$; stage 3.1 or 1.4 vs. 2.8 or 1.5; stiffness 10.4 or 8.7 vs. 8.1 or 7.0 (both $p<0.02$ ). Distribution of SNPs was as follows: rs12979860-CC $29 \%$, -CT 55\% and -TT 16\%; rs8099917-TT 50\%, -GT 43\%, and -GG 7\%; rs7270101-AA 78\%, -AC 20\%, and -CC 2\%; rs $1127354-C C$ 87\%, -CA 11\%, and -AA 2\%. Eighty-one (43\%) and 126 (82\%) patients with HCV 1 or 4 and HCV 2 or 3 respectively, reached an SVR. By multivariate analysis, the IL28B-wt was the strongest predictor of SVR rate; it was higher in rs12979860-CC and rs8099917 TT-patients. The combined ITPA variant, defined as ITPase deficiency, was strongly associated with a significant anemia-protection during therapy as shown in Table 1:

\begin{tabular}{lrrrrr}
\hline & Baseline & Week 4 & Week 12 & Week 24 & p-value \\
\hline HCV RNA viral load $\left(\log _{10} \mathrm{IU} / \mathrm{ml}\right)$ & & & & & \\
IL28B-wt & $6.1 \pm 0.7$ & $2.2 \pm 1.5$ & $1.3 \pm 0.9$ & $1.0 \pm 0.2$ & 0.001 \\
IL28B-variant & $5.9 \pm 1.0$ & $3.1 \pm 1.9$ & $2.5 \pm 1.9$ & $2.1 \pm 1.8$ & 0.001 \\
Hb level $(\mathrm{mg} / \mathrm{dl})$ & & & & & \\
ITPA-wt & $147.5 \pm 13.4$ & $121.0 \pm 18.5$ & $118.3 \pm 12.5$ & $112.1 \pm 13.3$ & 0.001 \\
ITPA-variant & $148.1 \pm 13.1$ & $139.1 \pm 14.4$ & $126.9 \pm 11.6$ & $121.2 \pm 9.5$ & 0.001 \\
\hline
\end{tabular}

Conclusions: GENEQUALITY IL28B-ITPA TYPE is a reliable and fast assay for IL28B and ITPA SNPs identification. The profile of IL28B and ITPA SNPs is associated with HCV RNA $<3.0 \log _{10} \mathrm{IU} / \mathrm{ml}$ and with $\mathrm{Hb}$ reduction $<30 \mathrm{mg} / \mathrm{dL}$ respectively, at week 4 . The test is useful for treatment strategy.

P3

\section{Frequency and types of $R H D$ gene alleles in serologically D-negative blood donors from Zagreb area: $R H D$ screening} - yes or no?

Dogić V. ${ }^{1}$, Hundrić-Hašpl Ž. ${ }^{2}$, Bingulac-Popović J. ${ }^{1}$, Babić I. ${ }^{1}$, Juraković-Lončar N. ${ }^{2}$, Herceg I. ${ }^{1}$, Jagnjić S. ${ }^{2}$, Balija M. ${ }^{3}$, Jukić I. ${ }^{3}$

${ }^{1}$ Department of Molecular Diagnostics, CITM, Zagreb, Croatia

${ }^{2}$ Department of Immunohematology, CITM, Zagreb, Croatia

${ }^{3}$ Transfusion Medicine Service, CITM, Zagreb, Croatia 
Background: $R H D$ gene changes cause reduced D antigen expression on the red blood cell (RBC) membrane (weak D, DEL). Individuals with DEL phenotype are always D-negative with standard serologic testing. They can only be identified serologically by additional adsorption and elution. Weak D, DEL, and D+/- chimera can indicate anti-D immunization.

Objectives: To assess the frequency and type of $R H D$ gene alleles causing different D variants (weak D, DEL) or D+/- chimera in a group of serologically D-negative blood donors using molecular $R H D$ screening.

Materials and methods: A total of 6523 samples obtained from D-negative donors were tested by indirect antiglobulin test for the presence of $R H D$ gene in pools of 20 samples. The qPCR method (RT-PCR System 7500, Applied Biosystems, USA) with TaqMan chemistry was used for exon 7 and exon 10 amplification. RHD genotyping of all qPCR-positive samples was performed to define RHD alleles using the PCR-SSP genotyping kit (Inno-Train, Kronberg, Germany).

Results: From 6523 samples, 23 (0.35\%) were found to be RHD-PCR positive. The allele RHD (M295I) was detected in 4/23 (17.4\%) and weak D type 2 in 2/23 (8.7\%) samples. The RHD-CE (2-9)D-CE large hybrid allele was found in 11/23 (47.8\%) samples. Carriers of this allele lack D antigen on the RBC membrane. Another 6 RHD-PCR-positive samples are scheduled for sequencing. All of the RHD-PCR-positive samples were also found to be C- and/or E-positive.

Conclusions: The frequency of clinically significant $R H D$ alleles in the population of serologically D-negative blood donors from Zagreb area is 1 out of 543. Upon completion of the study, we will decide on the introduction of molecular $R H D$ screening in routine testing of D-negative donors.

\section{P4}

\section{A retrospective study comparing the genetic test $\left(\mathrm{C} / \mathrm{T}_{-13910}\right.$ polymorphism) and combined hydrogen/methane breath test results of lactose malabsorption testing in daily clinical routine}

Enko D., Rezanka E., Stolba R., Baumann G.

Department of Laboratory Medicine, Central Hospital Steyr, Austria

Background: The primary lactose malabsorption is an inherited autosomal recessive form and results in a decline of lactase enzyme activity in the small intestine. A single nucleotide polymorphism $\left(\mathrm{C} / \mathrm{T}_{-13910}\right) 14 \mathrm{~kb}$ upstream from the lactase gene (LCT) locus is associated with this most common phenotype found in humans. The secondary lactose malabsorption is an acquired and reversible form associated to gastrointestinal diseases.

Objectives: To compare results obtained by the genetic test $\left(\mathrm{C} / \mathrm{T}_{-13910}\right.$ polymorphism) to those obtained by the hydrogen/methane $\left(\mathrm{H}_{2} / \mathrm{CH}_{4}\right)$ breath test in daily clinical routine.

Materials and methods: 263 outpatients were included in the study. The LCT C/ $\mathrm{T}_{-13910}$ polymorphism was performed using a melting curve analysis on the LightCycler Instrument (Roche Diagnostics, Rotkreuz, Switzerland). For gas-chromatography, the QuinTron Model DP Plus MicroLyzer ${ }^{\mathrm{TM}}$ (QuinTron, Milwaukee, Wisconsin, USA) was employed to measure the end-expiratory breath $\mathrm{H}_{2}$ and $\mathrm{CH}_{4}$ concentrations at 15, $30,45,60,75,90$, and 120 minutes after the ingestion of $50 \mathrm{~g}$ lactose. The breath test result was considered positive if the $\mathrm{H}_{2}$ and/or the $\mathrm{CH}_{4}$ peak were $>20 \mathrm{ppm}$ over the baseline value.

Results: Of 263 patients, 51 (19.4\%) had a C/C $\mathrm{C}_{-13910}$ genotype, indicating primary lactose malabsorption. Only 19 (7.2\%) patients had also a positive $\mathrm{H}_{2} / \mathrm{CH}_{4}$ breath test. $136(51.7 \%)$ patients had a C/T $\mathrm{T}_{-13910}$ and $76(28.9 \%)$ patients a $\mathrm{T} / \mathrm{T}_{-13910}$ genotype, indicating lactase persistence. Four (1.5\%) patients with the $\mathrm{C} / \mathrm{T}_{-13910}$ genotype and $1(0.4 \%)$ patient with the $\mathrm{T} / \mathrm{T}_{-13910}$ genotype had a positive $\mathrm{H}_{2} / \mathrm{CH}_{4}$ breath test result, indicating secondary lactose malabsorption. Cohen's Kappa between the two methods was 0.44 . When compared to the gold standard lactose breath test, the sensitivity of the genetic test was $79 \%$, the specificity $87 \%$, the positive predictive value $60 \%$, and the negative predictive value $98 \%$. Conclusions: Results obtained by this study revealed only moderate agreement between the genetic test and the breath test in daily clinical lactose malabsorption testing. Secondary lactose malabsorption as well as user-related pre-analytical problems such as the handling of the gas-chromatography instrument or instructing the patients to exhale end-expiratory breath may contribute to discrepant results. In daily clinical practice, both methods should be performed.

\section{P5}

\section{Hepatitis E exposure in blood donors in Upper Austria}

Fischer C., Hofmann M., Hofer K., Kaar J., Bartl I., Gabriel C. Red Cross Transfusion Service for Upper Austria, Linz, Austria

Background: In the last years, different studies showed that hepatitis E virus (HEV) is a growing public health concern in many developed countries. Therefore, HEV infections might bear a risk for transmission via blood transfusion and the clinical relevance of transfusion transmitted HEV infections still requires further investigation.

Objectives: To get an overview of acute HEV infections in Upper Austrian volunteer blood donors as well as occupational risk in transfusionrelated infections. 
Materials and methods: HEV RNA was extracted from $1 \mathrm{ml}$ EDTA plasma from pools of 96 blood donations by using the Magna Pure Compact Nucleic Acid Isolation Kit I (Roche Diagnostics). Amplification and detection was performed using the HEV RT-PCR Kit (altona Diagnostics) based on qPCR. Additionally, HEV-positive samples were characterized by sequencing the ORF2 region and sera were tested for anti-HEV by WanTai anti-HEV IgM and IgG assays.

Results: Between February 2013 and February 2014 a total of 48,704 individual blood donors were tested for HEV RNA. Five of these donors (0.01\%) were PCR-positive without pathological findings. All HEV RNA-positive donor samples were genotyped and revealed genotype 3 isolates. The HEV RNA concentrations determined by qPCR ranged from 2,217 to 293,635 IU/ml. None of the donors developed chronic disease. Follow-up serological testing revealed that none of the donors was seropositive for HEV IgM or IgG antibodies at the time of donation. Serum levels of ALT and CRP were within the normal range.

Conclusions: One out of 9,741 blood donations was found to be HEV RNA-positive in Upper Austria. Based on this data HEV-PCR screening is strongly recommended to prevent transmission of hepatitis E virus by transfusion.

P6

\title{
A survey on the adoption of reference materials by Brazilian laboratories performing nucleic acid testing for viral diagnosis and disease monitoring
}

\author{
Flatschart R.B., Venturelli P.N., Dias R.F., Granjeiro J.M. \\ National Institute for Metrology, Quality and Technology - INMETRO, Rio de Janeiro, Brazil
}

Background: In order to ensure reliability on the diagnosis and monitoring of infectious diseases, regulatory authorities around the world incorporated concepts regarding harmonization, traceability, and measurement uncertainty to their directives. Analytical results produced on real world samples with off-the-shelf diagnosis systems developed by any manufacturer can only reflect "true" values through the use of a common reference material characterized on a primary measurement system.

Objectives: To evaluate the use and acceptance of reference materials in private laboratories performing nucleic acid testing for viral diseases and in the public disease surveillance system.

Materials and methods: A questionnaire was distributed to 200 private members of the Brazilian Society for Clinical Pathology and Laboratory Medicine. The identical questionnaire was also sent to 11 public laboratories belonging to the disease surveillance system by the national regulatory agency.

Results: Of all laboratories responding, $61 \%$ did not use reference materials on qualitative or quantitative assay. $16 \%$ used them but conceptual confusion on the meaning of true reference materials and other materials accepted for quality control routinely was detected. Despite this, 30\% of laboratories showed interest in buying and using reference materials, irrespective of origin or expenses.

Conclusions: Reference materials for nucleic acid testing of viral diseases will have market acceptance in laboratory settings but some education is required to ensure its adequate application.

P7

\section{PCR-based rapid identification of pathogens in positive blood cultures using the FilmArray blood culture identifica- tion panel}

Leitner E. ${ }^{1}$, Klingsbigel S. ${ }^{1}$, Neuhold B. ${ }^{2}$, Hoenigl M. ${ }^{2}$, Feierl G. ${ }^{1}$, Krause R. ${ }^{2}$, Grisold A.J. ${ }^{1}$

${ }^{1}$ Bacteriology and Mycology Laboratory, IHMEM, Medical University of Graz, Austria

${ }^{2}$ Section of Infectious Disease and Tropical Medicine, Department of Internal Medicine, Medical University of Graz, Austria

Background: Sepsis is a life threatening medical condition caused by different infectious microorganisms. Culture-based methods represent the reference standard method for the detection and identification of pathogens, but they are time consuming and can be improved by using additional test systems. The novel FilmArray blood culture identification panel (BCID; Biofire Diagnostics) is a user-friendly multiplex PCR system offering sample preparation, amplification, and detection in one run with minimum hands-on-time.

Objectives: To evaluate the FilmArray BCID panel in positive blood cultures.

Materials and methods: Anonymized positive blood culture samples (BACTEC Plus Aerobic/F or BACTEC Plus Anaerobic/F blood culture bottles; Becton Dickinson Diagnostic Systems) were investigated after routine processing. Only one positive sample per patient was included in the study. The FilmArray BCID simultaneously detects the 27 most important bacterial and fungal pathogens and three resistance mechanisms (mecA, vanA/B, KPC). The blood culture samples were tested with the FilmArray BCID according to the manufacturer's instructions and cultured for identification and susceptibility testing in parallel. Identification was carried out with the VitekMS (bioMérieux) and susceptibility testing was done according to EUCAST guidelines.

Results: Eighty reported positive blood culture bottles were analyzed and compared to the reference standard blood culture. Blood culture results were as follows; $90 \%$ (72/80) positive blood cultures showed monomicrobial growth, 8.8\% (7/80) polymicrobial growth and one no growth, respectively. In the 72 monomicrobial samples 3 yeasts, 23 Gram-negative rods (including 52.2\% E. coli) and 46 Gram-positive 
microorganisms (including 42.5\% S. aureus) were identified. In the 7 polymicrobial samples, there was one mixture of Gram-positive and Gram-negative bacteria, 2 mixtures of Gram-positives, and 4 of Gram-negatives. Altogether, 90 microorganisms were detected in 79 samples. Concordant results for identification were achieved in 96.7\% (87/90) and for resistance genes in 100\% (10/10) with the most relevant detections of vancomycin resistant Enterococcus (VRE) and Candida glabrata in this study.

Conclusions: The FilmArray BCID panel is a rapid, easy to handle PCR system for the use in positive blood cultures without Gram-staining result. Integrated PCR for resistance genes and fungi identified a VRE and a Candida glabrata which may have an important impact on treatment decisions in septic patients.

\title{
P8
}

\section{Post-transfusion hepatitis B infection: Is donor screening for HBsAg and HBV DNA sufficient?}

\author{
Mayerhofer T. ${ }^{1}$, Höfinger A. ${ }^{2}$, Krucher H. ${ }^{2}$, Aliskanovic V. ${ }^{1}$, Trubert-Exinger D. ${ }^{1}$, Kessler H.H. ${ }^{3}$, Stelzl E. ${ }^{3}$, Haushofer A.C. ${ }^{1,4}$ \\ ${ }^{1}$ Institute of Laboratory Medicine, Landesklinikum St. Poelten, Austria \\ ${ }^{2}$ Blood Bank, Landesklinikum St. Poelten, Austria \\ ${ }^{3}$ Research Unit Molecular Diagnostics, IHMEM, Medical University of Graz, Austria \\ ${ }^{4}$ Institute of Medical and Chemical Laboratory Diagnostics with Blood Bank, Klinikum Wels-Grieskirchen, Wels, Austria
}

Background: In addition to mandatory donor screening for HBsAg, blood is also screened on HBV DNA in Austria. The serological pattern of anti-HBc antibody (HBcAb) positivity without HBs antigen (HBsAg) and anti-HBs antibody (HBsAb) positivity may be present in up to 4\% of Europeans. Serum HBV DNA can be detected in up to $10 \%$ of them, depending on the population studied.

Objectives: To demonstrate transmission of HBV through blood products from an HBsAg negative donor with a very low HBV load in blood. Materials and methods: In a look-back study, 11 of 22 patients who had received erythrocyte concentrates between 2002 and 2010 from an HBsAg negative donor who was found to be positive for HBV DNA subsequently were investigated for the post transfusion hepatitis B status. Transfusion recipients were tested on HBsAg, HBcAb, and HBsAb using the Architect (Abbott, Wiesbaden, Germany) and the Ortho ECI (Ortho Clinical Diagnostics, London, UK) platforms. The serum HBV DNA load was tested by the Cobas AmpliPrep/Cobas Taqman (Roche Molecular Systems, Branchburg, NJ) assay. Sequencing of HBV was done by Sanger sequencing using the TRUGENE HBV Genotyping Kit (Siemens Siemens Healthcare Diagnostics, Tarrytown, NY). Up to 2011, donors had been screened by a qualified pool HBV PCR followed by a commercial available PCR system, the Cobas S201 (Roche).

Results: Of 11 patients, 6 did not give evidence to a serological reaction regarding HBV infection with HBV DNA being undetectable, 4 showed a serology according to past hepatitis B infection (3 of them post transfusion) with undetectable HBV DNA), and one showed HBsAg only. One of the patients had accidently been investigated on HBV DNA in 2010 and an HBV DNA load of $30500 \mathrm{IU} / \mathrm{ml}$ had been found. When the donor was retested (retain and fresh samples) with the Cobas S201 system and the Cobas AmpliPrep/Cobas Taqman assay, HBV DNA could always be detected $(<20 \mathrm{IU} / \mathrm{ml})$ and $\mathrm{HBcAb}$ were found. When sequences obtained from the donor's and the recipient's HBV strains were compared, both showed HBV genotype D and the alignment analysis of $>509$ nucleotides revealed two heterologies only.

Conclusions: Alignment analysis showed that at least one of the patients was most likely infected through an erythrocyte concentrate that was derived from an HBsAg negative donor with a very low HBV DNA load in blood. With introduction of the latest version of systems, the Cobas S201, including smaller pools (pools of 6 samples), HBV DNA became detectable in the sample. Testing on HBcAb may be considered as an additional valuable screening tool for identifying an HBsAg negative donor with a very low HBV DNA concentration in blood.

\section{P9}

\section{Amino acid substitutions in Hepatitis C virus core protein are associated with advanced liver disease and hepatocel- lular carcinoma}

Mohar B. ${ }^{1}$, Milić S. ${ }^{2}$, Čarapina M. ${ }^{3}$, Katunarić M. ${ }^{1}$, Štimac S. ${ }^{2}$, Grahovac B. ${ }^{1}$

${ }^{1}$ Department of Pathology, Medical Faculty University of Rijeka, Croatia

${ }^{2}$ Internal Clinic, Clinical Hospital Center Rijeka and Medical Faculty University of Rijeka, Croatia

${ }^{3}$ Department of Pathology, General Hospital Zabok, Croatia

Background: Hepatitis C virus (HCV) is the main cause of chronic hepatitis, cirrhosis, and hepatocellular carcinoma (HCC) worldwide. Several $\mathrm{HCV}$ proteins can alter cellular growth and the core protein is the most strongly associated with cellular transformation. HCV core gene mutations and subsequent amino acid changes in the core protein were reported to be associated with advanced liver disease and increased HCC risk.

Objectives: To determine mutations in the HCV core gene at diagnosis and to investigate a possible relationship between specific mutations and subsequent course of liver disease and patient outcome.

Materials and methods: HCV RNA was isolated from serum samples of 13 patients with chronic HCV infection that had been collected between 2006 and 2009. Viral RNA was transcribed to cDNA and the entire HCV core gene was amplified by nested PCR. Amplification products were 
sequenced with the Big Dye Termination v1.1 method on an ABI 310 sequencer. The HCV core sequence data obtained was compared with reference HCV genotype sequences from GenBank.

Results: Six patients were infected with HCV subtype 1b, five patients with 1a, and 2 patients with 3a. Two of the six patients with HCV subtype $1 \mathrm{~b}$ showed progressive liver disease and another two developed HCC. In all patients, mutations R70Q/H and T75A were found; HCC patients showed mutations B140L and S106N additionally. One of patients with progressive liver disease showed the mutation M91L additionally. Of the five patients with subtype 1a and early virological response showed a core region identical to the reference HCV subtype 1a from GenBank. The remaining patients with subtype 1a and sustained virological response showed 1 to 4 mutations located at the 3'end of the core gene. In addition, sequence analyses demonstrated 10 to 20 sense mutations in all samples and all HCV subtypes, corresponding to the high mutation rate of $\mathrm{HCV}$.

Conclusions: Preliminary data suggests that the presence of specific mutations at diagnosis may serve as a prognostic marker for the subsequent course of disease and patient outcome.

\section{P10}

\section{Evaluation of the Bio-Rad Dx Real-Time System in combination with the MagNA Pure platform (Roche) for the detection of Mycoplasma genitalium in urogenital specimens}

Vanmassenhove B., Alliet G.

Clinical laboratory, Az Damiaan, Ostend, Belgium

Background: Mycoplasma genitalium (MG) can cause urogenital infection.

Objectives: To compare the performance of the Bio-Rad Dx CT/NG/MG Assay for the detection of MG with that of a validated in-house method. Materials and methods: DNA was extracted using the MagNA Pure platform (DNA I High Performance protocol). $200 \mu \mathrm{l}$ of sample was used for extraction. Elution was done in $110 \mu \mathrm{l}$ buffer. $5 \mu \mathrm{l}$ of the eluate was used for qPCR. The assay was checked for accuracy, linearity, analytical sensitivity, and precision.

Results: Accuracy: 73 samples were tested. 5 out of 37 positive samples were missed by the Dx Real-Time System. All 5 had a high Cp value with the in-house method. The total agreement was 93\%. Linearity: The AmpliRun ${ }^{\circledR}$ DNA control was diluted serially. The slope was -3.47 (efficiency: 98.7\%). Analytical sensitivity: A negative PreservCyt specimen was spiked with an AmpliRun ${ }^{\circledR}$ MG DNA control (Vircell) to determine the limit of detection (LOD with a 95\% hit rate). The LOD was found to be $5000 \mathrm{copies} / \mathrm{ml}$, which was about 10 times higher than stated in the package insert. The LOD of the in-house test is about 4000 copies $/ \mathrm{ml}$. Precision: One positive and one weak positive sample were extracted in triplicate on 3 different days. All 9 results were positive. The standard deviation and the coefficient of variation were similar as described in the package insert.

Conclusions: The Bio-Rad Dx Assay is significantly less sensitive than our in-house method; however, it is an easy method with few hands on time and can detect 3 pathogens simultaneously in one reaction (Chlamydia trachomatis, Neisseria gonorrhoeae, and MG). The assay met our validation criteria. As the lysis step proposed in the instructions for use is time consuming, the MagNA Pure platform offers a good alternative.

P11

\section{Daily microbiological screening for detection of catheter-related bloodstream infections in hematooncological patients}

Wagner J. ${ }^{1}$, Duettmann W. ${ }^{2}$, Theiler G. ${ }^{2}$, Neumeister P. ${ }^{2}$, Zollner-Schwetz I. ${ }^{1}$, Hoenigl M. ${ }^{1}$, Valentin T. ${ }^{1}$, Rabensteiner D. ${ }^{1}$, Krause R. ${ }^{1}$ ${ }^{1}$ Section of Infectious Diseases and Tropical Medicine, Department of Internal Medicine, Medical University of Graz, Austria ${ }^{2}$ Division of Hematology, Department of Internal Medicine, Medical University of Graz, Austria

Background: Currently, diagnostic tests for catheter-related bloodstream infections (CRBSI) are performed in patients with clinical suspicion (i.e. fever, hypotension, chills, leukocytosis, and no other obvious focus of infection).

objectives: To evaluate if daily screening using the peptide nucleic acid (PNA) FISH and acridine orange leucocyte cytospin (AOLC) tests might provide an earlier detection of CRBSI in a subclinical stage in hemato-oncological patients with central venous catheters (CVCs) in place.

Materials and methods: 175 hemato-oncological patients with 325 catheter periods were investigated prospectively. Daily, 3 mL of EDTA blood was drawn from the distal catheter lumen of the CVC prior to routine blood sampling. Screening for CRBSI was performed by PNA FISH test with commercially available universal hybridization probes (for detection of all relevant and potentially pathogenic bacteria and fungi) and compared to the AOLC test; culture on chocolate agar served as control. If CRBSI was clinically suspected, routine investigation was performed by routine Gram stain/AOLC tests, differential time to positivity (DTP) and, if the catheter was pulled, Brun-Buisson-method at the discretion of the treating physician blinded to the screening result. 
Results: Thirty-three patients had a double lumen, 183 a triple lumen, and 4 a five-lumen CVC. 105 patients had an implanted port (33 single and 72 double chambers). 4594 blood samples were investigated. 17 CRBSIs were detected in 5807 catheter days by routine measures resulting in a CRBSI rate of 2.9/1000 catheter days. Two CRBSIs could be anticipated by positive PNA FISH test and 5 by positive AOLC screenings. In 11 CRBSI cases screening was negative. In 10 catheter periods, screening was positive but patients had no subsequent signs or symptoms indicative for CRBSI. Following no routine diagnostic measure for detection of CRBSI was ordered and the patients were considered not to have CRBSI. The PNA FISH screening showed a sensitivity of 12\%, specificity of 98\%, PPV of 22\%, and NPV of 95\%; the AOLC test showed a sensitivity of $29 \%$, specificity of $97 \%$, PPV of $33 \%$, and NPV of $96 \%$.

Conclusions: Screening for CRBSI in hemato-oncological patients does not seem to be a useful and cost-efficient tool for anticipative diagnosis of CRBSI. Reasons for false negative results might include dilution of microbial burden due to concomitant administered infusions during sampling, and origin of CRBSIs from the alternative lumens not sampled for screening. False positive results might origin from catheter colonization without subsequent spread of microorganisms into the peripheral bloodstream. 


\section{Author Index}

\author{
Akoua-Koffi C. A16 \\ Alanio A. A5 \\ Aliskanovic V. P8 \\ Alliet G. P10 \\ Asthana D. A7, A25 \\ Avettand-Fenoel V. A24 \\ Babic I. P1, P3 \\ Balija M. P1, P3 \\ Balsat M. A5 \\ Bartl I. P5 \\ Baumann G. P4 \\ Beck R. A24 \\ Bingulac-Popovic J. P1, P3 \\ Bolivar H. A7 \\ Bortolozzo K. P2 \\ Bozic, M. A10 \\ Bugin E. P2 \\ Carapina M. P9 \\ Cavalletto L. P2 \\ Chemello L. P2 \\ Chudy M. A18 \\ Ciotti M. A20 \\ Cloherty G.A. A7 \\ De Boever P. A16 \\ Diaz R.F. P6 \\ Di Lorenzo C. A1 \\ Dolan A. A3 \\ Dogic V. P1, P3 \\ Duettmann W. P11 \\ Enko D. P4 \\ Esser S. A15 \\ Estambale B. A16 \\ Ftkenheuer G. A15 \\ Feierl G. P7
}

Fischer C. P5

Flatschart R.B. P6

Freeman-Cook L.L. A9

Frishman D. A24

Gabriel C. A19, P5

Ginocchio C.C. A2, A23

Goswami N. A16

Grahovac B. P9

Granjeiro J.M. P6

Graziadei I. A13

Greffrath U. A11

Grisold A.J. P7

Haase K. A24

Harrel B.T. A9

Hartmann A. A10

Haushofer A.C. P8

Heckmann M. A11

Herceg I. P3

Hess M. A12

Hoenigl M. P7, P11

Hofer K. P5

Hfinger A. P8

Hoffmann D. A24

Hofmann M. P5

Hundric-Haspl Z. P1, P3

Hussenet C. A5

Ikenberg H. A21

Izopet J. A4, A14

Jagnjic S. P3

Jensen B. A15

Jukic I. P1, P3

Jurakovic-Loncar N. P1, P3

Kaar J. P5

Kaiser R. A15
Katunaric M. P9

Kessler H.H. A10, A16, P8

Klingsbigel S. P7

Knoop D. A10

Knig B. A6

Krause R. P7, P11

Kress J. A18

Krucher H. P8

Lafaurie-Bergeron A. A5

Leatham B.R. A9

Le-Goff J. A4, A5

Leitner E. P7

Lengauer T. A15

Lbke N. A15

Mansuy J.-M. A4

Matthiesen P. A10

Mayerhofer T. P8

Mengelle C. A4

Mercier-Delarue S. A4, A5

Milic S. P9

Miskovic B. P1

Mohar B. P9

Mratinovic-Mikulandra J. P1

Nawrot T. A16

Neuhold B. P7

Neumeister P. P11

Niesters H.G.M. A1

Nbling C.M. A17, A18

Oette M. A15

Picard-Maureau M. A5

Protzer U. A24

Rabensteiner D. P11

Rathore M. A25
Rezanka E. P4

Rottengatter K. A12

Saune K. A4

Schiavo I. P2

Schroeder A.R.W. A9

Schlter E. A15

Simon C. A8

Simon F. A4, A5

Smith E. A8

Steimer M. A11

Stelzl E. A16, P8

Stimac S. P9

Stolba R. P4

Strijdom H. A16

Tabrizi S.N. A22

Tiemann C. A10

Theiler G. P11

Toth M. A7, A25

Trubert-Exinger D. P8

Tyler E.M. A9

Valentin T. P11

Valiathan R. A7, A25

Van Loon A.M. A1

Vanmassenhove B. P10

Van Sickle R.P. A9

Venturelli P.N. P6

Verine J. A5

Wagner J. P11

Wallace P.S. A1

Young T.P. A7

Zollner-Schwetz I. P11

Zuber J. A24 